ANIMAL BEHAVIOUR, 2006, 71, 773-779

doi:10.1016/j.anbehav.2005.05.014

Available online at www.sciencedirect.com

SCIENCE DIRECT

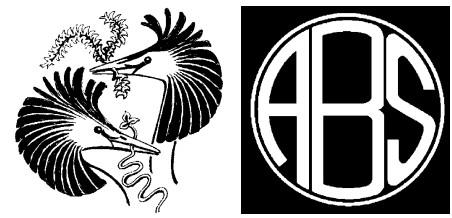

\title{
Wax combs mediate nestmate recognition by guard honeybees
}

\author{
PATRIZIA D'ETTORRE*, TOM WENSELEERS $\dagger$, JENNY DAWSON $\ddagger$, STUART HUTCHINSON $\ddagger$ \\ TOM BOSWELL $¥ \&$ FRANCIS L. W. RATNIEKS $\ddagger$ \\ ${ }^{*}$ LS Biologie I, Universität Regensburg, Germany \\ $\dagger$ Laboratory of Entomology, Zoological Institute, University of Leuven \\ $\ddagger$ Laboratory of Apiculture \& Social Insects, Department of Animal and Plant Sciences, \\ University of Sheffield
}

(Received 12 February 2005; initial acceptance 25 March 2005;

final acceptance 16 May 2005; published online 10 February 2006; MS. number: 8465)

\begin{abstract}
Research has shown that the wax combs are important in the acquisition of colony odour in the honeybee, Apis mellifera. However, many of these studies were conducted in the laboratory or under artificial conditions. We investigated the role of the wax combs in nestmate recognition in the natural context of bees at colony entrances. Wax combs constructed by each experimental colony were swapped between hives and the acceptance of nestmate and non-nestmate forager workers was recorded before and after the swap, and in relation to a control hive not involved in the swap. We conducted the experiment twice, in consecutive years with three different colonies each time. Colonies that exchanged combs became more accepting of each other's workers than of workers from the third colony. The overall acceptance of comb swap non-nestmates increased significantly from 3\% before the swap to $23 \%$ after the swap in the first year, and from 8 to $47 \%$ in the second year. This effect wore off within 3 weeks. Chemical analyses showed that the cuticular profile of non-nestmates involved in the experimental comb swap became more similar to each other after the swap, and that acceptance by guards of bees from different hives was negatively correlated with chemical distance between the hydrocarbon profiles of the workers from different hives.
\end{abstract}

(ㄷ) 2006 The Association for the Study of Animal Behaviour. Published by Elsevier Ltd. All rights reserved.

The ability to make adaptive recognition decisions is widespread in biological systems. Examples include the vertebrate immune system rejecting nonself tissues as a means of defence against parasites, a plant stigma rejecting self-produced pollen, or a female animal rejecting males of the wrong species. Recognition also occurs at the group level. Insect societies typically defend their nest entrance against harmful intruders. These include parasites and predators of different species, and also conspecific intruders from other colonies. Recognizing conspecific intruders is a particular challenge because

Correspondence and present address: P. D'Ettorre, Department of Population Biology, Institute of Biology, University of Copenhagen, Universitetsparken 15,2100 Copenhagen, Denmark (email: pdettorre@bi.ku. dk). T. Wenseleers is at the Laboratory of Entomology, Zoological Institute, University of Leuven, Naamsestraat 59, 3000 Leuven, Belgium. J. Dawson, S. Hutchinson, T. Boswell and F. L. W. Ratnieks are at the Laboratory of Apiculture \& Social Insects, Department of Animal and Plant Sciences, University of Sheffield, Sheffield S10 2TN, U.K. members of the same species will share many characteristics, including recognition cues (Sherman et al. 1997).

The honeybee, Apis mellifera, is an important model system for studying nestmate recognition and its underlying mechanisms (Breed 1998). Honeybee colonies frequently steal honey from each other, and this can lead to the death of the victimized colony. As such, the recognition of conspecific intruders is important. Acceptance occurs when the label of an incoming individual sufficiently matches the internal template of a discriminating individual (Crozier \& Pamilo 1996; Gamboa 2004). In a perfect recognition system, a guard would be able to accept all nestmates and reject all non-nestmates (cf. Sherman et al. 1997). However, when nestmates and non-nestmates have overlapping recognition cues, recognition errors will occur (Reeve 1989).

Several studies have investigated the mechanism underlying the ontogeny of nestmate recognition in the honeybee. Newly emerged worker honeybees carry no recognition cues, and so present a 'blank state' facilitating their acceptance into their own colony before acquiring 
the colony-specific recognition phenotype (Breed et al. 2004a). Exposure to nest material is an essential step in the acquisition of colony odour of many species of social insects, including paper wasps (e.g. Gamboa 2004) and social parasites. For example, in Polistes, usurping queens apply their odour to the comb and their acceptance by emerging host workers probably depends on the similarity of odour between the comb and the usurper (Cervo \& Lorenzi 1996). Honeybee nests comprise a series of parallel vertical wax combs which are built from wax secreted by the workers. Laboratory experiments have shown that young bees acquire recognition cues through contact with the comb (Breed et al. 1995; Breed 1998). Wax comb consists predominantly of fatty acids (Breed 1998) but hydrocarbons are also present (Fröhlich et al. 2000; Wakonigg et al. 2000). Chemical cues acquired from the comb can also be transferred among bees that are in physical contact, resulting in a homogeneous colony odour (Breed et al. 1995).

Although the evidence for the role of the wax comb in nestmate recognition is convincing, it comes primarily from laboratory studies using newly emerged bees and frequently from encounters that are staged in small containers. We therefore investigated the effect of honeybee wax combs on the acceptance of conspecific nestmate and non-nestmate workers at the hive entrance by naturally occurring guard workers. We allowed three colonies to build their own combs and determined the acceptance of nestmates and non-nestmates; we then swapped half the wax combs between two of the colonies. The experiment was repeated with three more colonies the following year.

\section{METHODS}

\section{Study Species and Experimental Set-up}

Six queenright honeybee colonies of mixed European race, but predominantly Apis $m$. mellifera, were studied. The colonies had approximately equal numbers of workers (10 000-20 000) and were housed in standard Langstroth hives with nine frames, including two to four frames of brood. In the 2-month period before our behavioural observations, each colony had been allowed to build its own wax combs: we gradually removed the existing frames and replaced them with empty frames in which the colonies built new combs. Colonies received sucrose syrup to encourage comb building. We conducted the study at the Laboratory of Apiculture and Social Insects, Sheffield, U.K. Three colonies (A, B, C) were studied during October and November 2002 and three colonies (D, E, F) during October and November 2003. The three colonies were arranged in a row with a large (ca. 3-m) gap between neighbouring hives to minimize drifting of foragers. Each hive had a circular entrance hole $3.5 \mathrm{~cm}$ in diameter, below which a wooden platform $(20 \times 10 \mathrm{~cm})$ was attached to facilitate the introduction and observation of bees. We placed experimentally introduced bees on this platform where the guards would find them. We did the experiment only on days when the temperature was above $10^{\circ} \mathrm{C}$ and the bees were active and flying from the colonies. Heat lamps $(500 \mathrm{~W})$ were used to warm the nest entrance so that guards would patrol the entrance platform even on cool days.

\section{Bioassays of Acceptance of Foragers by Guards}

We used a standard assay of guard discrimination (Downs \& Ratnieks 2000). Returning forager workers without pollen were captured at the entrance of their own colony (the source colony), individually placed in plastic vials and cooled in a portable ice chest. The chilled bees were then allowed to warm up before being released individually at the entrance of a discriminator colony. The chilling made the bees reluctant to fly away even though they were fully active on release. We observed the reaction of guards for $5 \mathrm{~min}$. An introduced worker that was bitten, stung or dragged by guard bees was classed as rejected. Workers that were only antennated or licked by guards or that remained at the entrance for $5 \mathrm{~min}$ without receiving aggression were classed as accepted. Introductions were made by a pair of researchers, one of whom acted as the observer and was blind with respect to the colony of origin of the introduced bee.

To introduce bees, we collected three returning foragers from one source hive. One was introduced back to its own hive (nestmates) and one each into the other two hives (non-nestmates). The procedure was repeated with foragers from the other two hives to constitute one round of nine introductions. We made 4-13 rounds of introductions per day depending on the duration of suitable weather.

After several days of experimental introductions into unmanipulated colonies over a period of ca. 2 weeks, we reciprocally swapped five frames of wax combs without brood between two of the three hives (B and C in 2002 and $\mathrm{E}$ and $\mathrm{F}$ in 2003) and placed them so that they alternated with the colony's own combs. In this way the cluster of bees, which covered four to six combs, could not be located on combs originating from only one of the two colonies. The third hive (A in 2002 and D in 2003) received a control manipulation consisting of the removal and replacement of five of its own frames. We made the manipulation at the end of the day so that the hives could settle before the next day of data collection began. After the comb swap we made six to eight rounds of introduction per day over approximately 3 weeks. No other hive manipulations or inspections were made during the datagathering period.

In 2002, 297 bees were introduced before the comb swap and 360 bees up to 17 days after the swap. In 2003, 378 bees were introduced before the comb swap and 801 up to 23 days after the swap.

\section{Chemical Analysis of Workers}

In 2003, we analysed the cuticular profile of worker bees (returning forager workers without pollen) from the three experimental hives before and after the swap. Workers 
were frozen in individual glass vials and we extracted surface chemicals by washing each bee in $500 \mu$ l of pentane for $10 \mathrm{~min}$. We injected $2 \mu \mathrm{l}$ of this extract into an Agilent Technologies $6890 \mathrm{~N}$ gas chromatograph with a flame ionization detector, equipped with a capillary column (Rtx-5, $30 \mathrm{~m} \times 0.25 \mathrm{~mm} \times 0.50 \mu \mathrm{m}$, Restek, Bellefonte, PA, U.S.A.). The injector was a split-splitless type, the carrying gas helium at $1 \mathrm{ml} / \mathrm{min}$, and the temperature rose from $70^{\circ} \mathrm{C}$ to $280^{\circ} \mathrm{C}$ at $10^{\circ} \mathrm{C} / \mathrm{min}$, then from $280^{\circ} \mathrm{C}$ to $310^{\circ} \mathrm{C}$ at $3{ }^{\circ} \mathrm{C} / \mathrm{min}$, and it was held at $310^{\circ} \mathrm{C}$ for $10 \mathrm{~min}$. Compounds were identified on the basis of their mass spectra from electron ionization mass spectrometry using a Hewlett Packard 5890A gas chromatograph coupled to an HP 5917A mass selective detector $(70 \mathrm{eV}$ electron impact ionization) and comparison with standards.

\section{Statistical Analysis}

We used a generalized linear model (GLZ) with binomial error structure and logit link function (Neter et al. 1996) to test for the effect of wax comb on nestmate recognition. This was done by (1) comparing the acceptance of introduced non-nestmate bees before and after the comb swap (e.g. acceptance of C colony bees by colony B before and after the comb swap) or (2) comparing the acceptance of non-nestmates after the comb swap from colonies with swapped versus nonswapped comb (e.g. acceptance of $\mathrm{C}$ versus $A$ bees by colony B). For comparison 1 we used the pooled data from 5 days before and 5 days after the comb swap and for comparison 2 we used all data collected after the comb swaps (Table 1). In all cases, the dependent variable was whether an introduced bee was accepted or not. To make sure that differences in the acceptance of non-nestmates before and after the comb swap were not merely caused by seasonal changes in the level of aggression of the bees, we also compared the

Table 1. Generalized linear model to test the effect of swapping wax combs between colonies on nestmate recognition

\begin{tabular}{|c|c|c|c|}
\hline & $d f$ & $\begin{array}{c}W / \log \\
\text { likelihood }\end{array}$ & $P$ \\
\hline \multicolumn{4}{|l|}{ Before/after swap* } \\
\hline DISCR & 3 & 10.46 & 0.015 \\
\hline BEFORE/AFTER & 1 & 19.62 & $<0.001$ \\
\hline DISCR*BEFORE/AFTER & 3 & 0.86 & 0.840 \\
\hline \multicolumn{4}{|l|}{ Swapped/nonswapped $\dagger$} \\
\hline DISCR & 3 & -239.94 & 0.002 \\
\hline SWAP/NONSWAP & 1 & -222.82 & $<0.001$ \\
\hline DISCR*SWAP/NONSWAP & 2 & -219.16 & 0.026 \\
\hline
\end{tabular}

The dependent variable was whether any one bee was accepted or not. A binomial error structure was used with a logit-link function. The independent variables were: DISCR: discriminator colony used; BEFORE/AFTER: before versus after comb swap; SWAP/NONSWAP: non-nestmates from colonies with swapped versus nonswapped comb. Analysis based on combined data from 2002 and 2003.

*Based on pooled data from 5 days before and 5 days after comb swap; Wald statistic given.

†Based on all data collected after comb swap; log-likelihood statistic given. acceptance of introduced non-nestmate bees from the colonies not involved in the comb swap (A and D) by colonies B, C, E and F. Data from all colonies studied in both years were combined in a single analysis, but all observed differences were also significant when the data from the 2 years were tested separately.

To analyse the chemical data, we determined the proportions of 16 identified hydrocarbons from the peak areas of each bee's profile. We used a multivariate statistical analysis to estimate the similarity of the chemical profiles of workers from different colonies before and after the comb swap. Peaks were first analysed with principal components analysis to reduce the number of variables subsequently used in a discriminant analysis (DA). We used a DA to determine whether predefined groups could be discriminated on the basis of their chemical profiles and to assess the degree of similarity among groups. The correct classification of individuals to the respective groups was verified. For all statistical analyses we used Statsoft Statistica 6.0 for Windows (StatSoft Inc., Tulsa, OK, U.S.A.).

\section{RESULTS}

\section{Bioassays}

In both years guard bees behaved as expected before the swap, accepting most nestmates and rejecting most nonnestmates (with some fluctuations in the control hive A in 2002). After the comb swap, there was a significant increase in acceptance of non-nestmates in the pair of hives involved in the comb swap (B/C and E/F; GLZ: factor BEFORE/AFTER: $P<0.001$; Table 1, Fig. 1). This effect was consistent across all four discriminator colonies used (DISCR), as shown by the nonsignificant first-order interaction effect DISCR*BEFORE/AFTER (Table 1). The observed effect was not due to a seasonal decrease in the level of acceptance by guards, as there was no significant increase in the acceptance of non-nestmate bees from control colonies $\mathrm{A}$ and $\mathrm{D}$, which were not involved in the comb swap (GLZ: $W=0.00002, P=0.99$; Fig. 1). Data collected after the combs were swapped showed that non-nestmate bees from colonies that had received swapped comb were accepted more frequently than nonnestmate bees from control colonies that had not received swapped comb (GLZ: factor SWAP/NONSWAP: $P<0.001$; Table 1). This effect was again consistent across all discriminator colonies used, although the magnitude of the effect varied significantly between colonies (GLZ: factor DISCR ${ }^{*}$ SWAP/NONSWAP: $P=0.026$ ).

The reciprocal acceptance of comb-swapped nonnestmates was significantly greater than the acceptance of control non-nestmates in the week after the comb swap but decreased with time and disappeared after 3 weeks (Fig. 2). That the effect of the comb swap wears off with time was confirmed with a generalized linear model (GLZ: DAY(DISCR): log likelihood $=-134.24, P<0.001$ ). In the first year the swap effect diminished more quickly than in the second year, but it was significant in both years. 
(a)

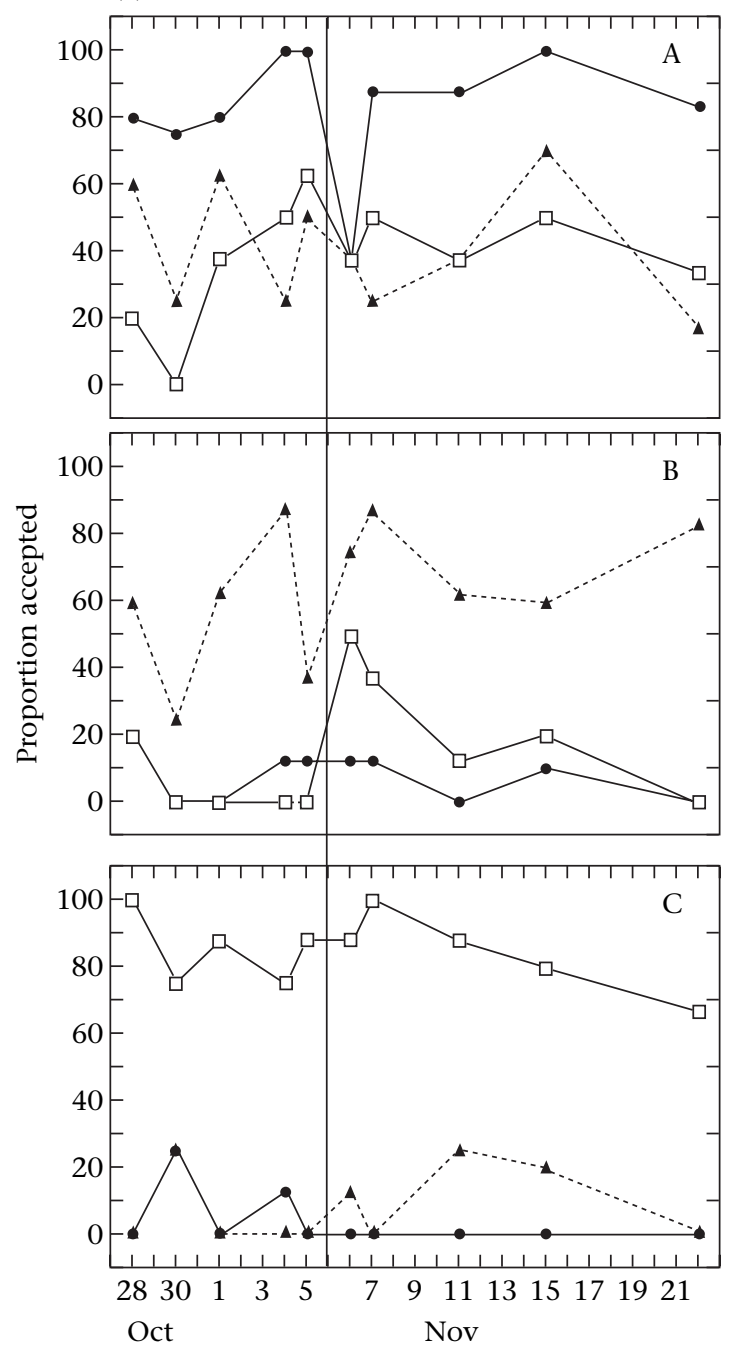

(b)

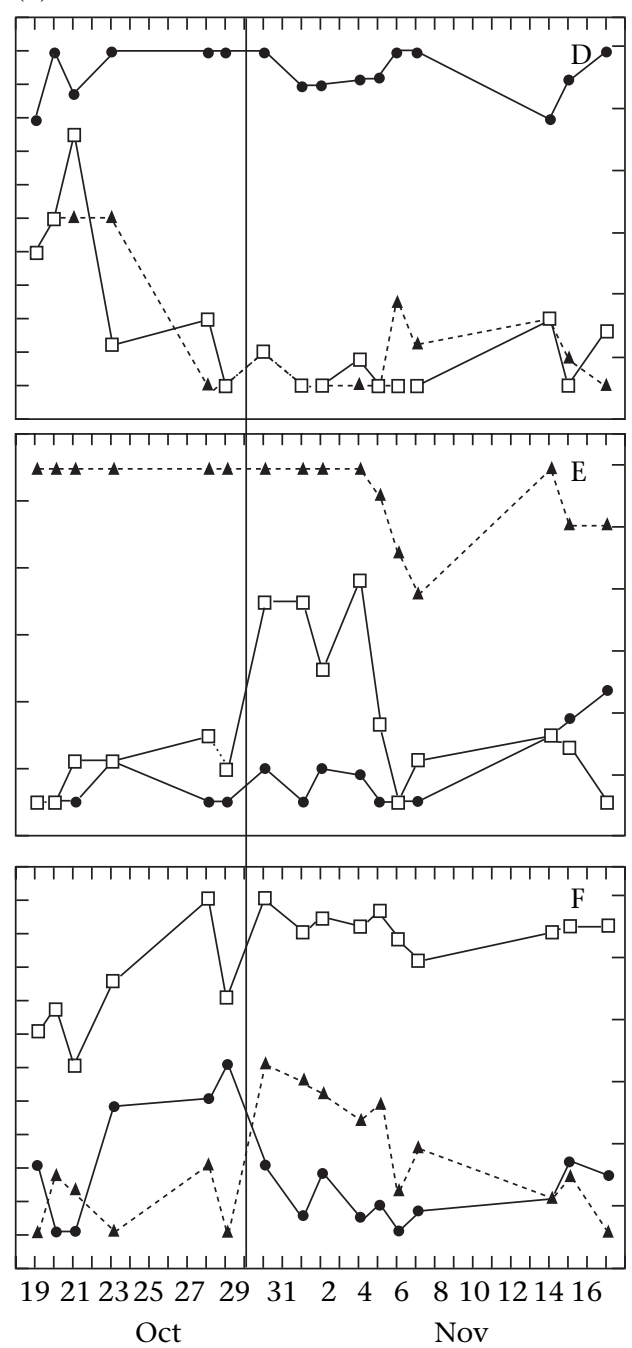

Date

Figure 1. Proportion of introduced bees accepted by guards in three colonies in (a) 2002 and three in (b) 2003. Wax combs were swapped between hives B and C on 5 November 2002 and between hives $E$ and F on 29 October 2003, at the end of the day (indicated by vertical lines).

- : Control hives (A in 2002 and D in 2003); $\mathbf{\Delta}$ : hives B (2002) and E (2003); $\square$ : hives C (2002) and F (2003).

\section{Chemical Analysis}

We confirmed that the hydrocarbons present on the cuticle of honeybee workers were represented mostly by a series of linear alkanes with chain lengths ranging from $\mathrm{C}_{23}$ to $\mathrm{C}_{33}$ and the corresponding alkenes (see Page et al. 1991; Wakonigg et al. 2000; Martin et al. 2001; Dani et al. 2004). Figure 3 shows the results of a statistical analysis of the chemical profiles of workers from colonies D, E and $\mathrm{F}$ before and after the swap. Discriminant analysis explained $89 \%$ of the total variance and significantly separated the six groups (Wilk's $\lambda=0.054, F_{25,75}=3.63$, $P<0.0001)$. Although the profiles of the three colonies were relatively similar before the comb swap, colony D diverged significantly whereas colonies $\mathrm{E}$ and $\mathrm{F}$, which received each other's combs, converged after the swap. The Mahalanobis distance between group centroids, which is a measure of the chemical distances between colonies, was comparable and not significantly different between colonies before the swap (Fig. 4a), but significantly shorter between $\mathrm{E}$ and F after the swap (Fig. 4b).

Combining the chemical data and the acceptance data, there was a highly significant negative correlation between the proportion of bees accepted by guards and the chemical difference in chemical profile between the guards' colony and the colony from which the introduced bees were taken (Mahalanobis distance in DA; Spearman correlation: $r_{\mathrm{S}}=-0.84, N=18, P<0.001$; Fig. 5).

\section{DISCUSSION}

Swapping combs between honeybee colonies had a clear effect on nestmate recognition: in both years the colonies with each other's combs became significantly more accepting of each other's bees than of bees from the control 


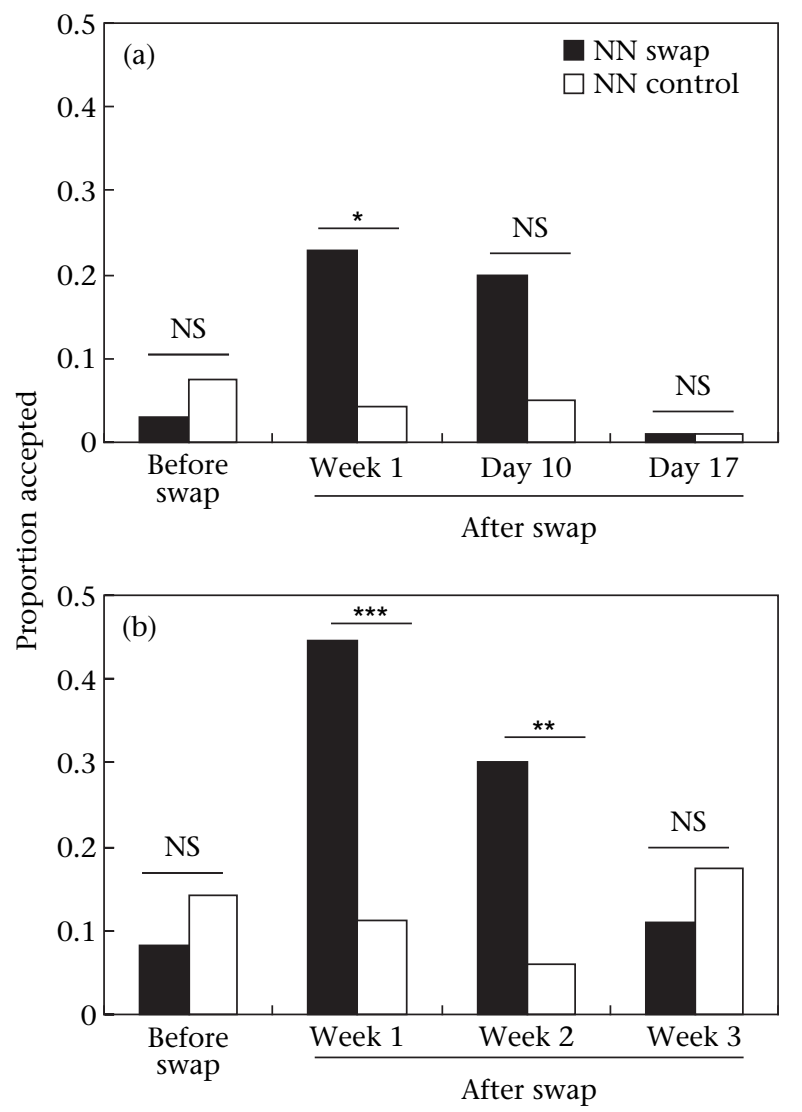

Figure 2. Proportion of swap non-nestmates (NN swap) and control non-nestmates (NN control) accepted by guards in (a) hives $B$ plus C (2002) and (b) hives E plus $F(2003)$. Wax combs were swapped between hives $B$ and $C$ in 2002 and between hives $E$ and $\mathrm{F}$ in 2003. ${ }^{*} P<0.05$; ${ }^{* *} P<0.01$; ${ }^{* *} P<0.001$ : generalized linear model (Table 1).

colony not involved in the comb swap. Chemical analyses showed that the cuticular profile of bees varied in accordance with the behavioural results: greater chemical similarities resulted in greater acceptance. Thus, our results clearly show that the wax combs of honeybee colonies provide cues that are used in nestmate recognition by guard bees. Even though the comb swap non-nestmates were not accepted as frequently as nestmates, the effect was strong. For example, the overall acceptance of comb swap non-nestmates increased from $3 \%$ before the swap to $23 \%$ after the swap in 2002 (Fig. 2a) and from 8 to $47 \%$ in 2003 (Fig. 2b). Nevertheless, the effect wore off within 3 weeks suggesting that recognition cues are constantly updated. These results are in agreement with research showing that acceptance between two parts of a colony that had been divided, as occurs in swarming, diverges with time (Beekman et al. 2002).

The cuticular profile of bees from the two hives involved in the reciprocal comb swap converged, whereas that of bees from the control nonswap hive diverged. This implies that at least some of the odour cues used in nestmate recognition come from the surface of wax combs and are continuously integrated into the insects' cuticles. This conclusion is strengthened by the correlation between the proportion of accepted bees and the chemical distance

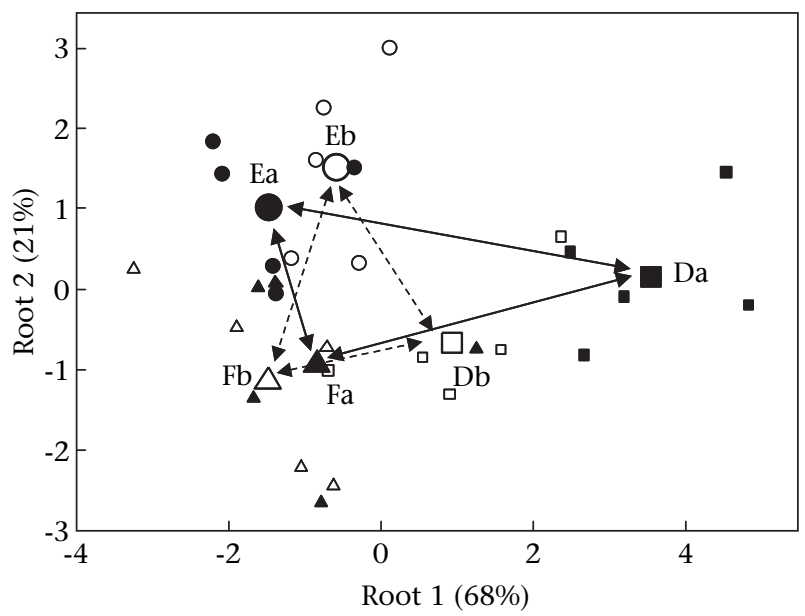

Figure 3. Plot of the first two functions of the discriminant analysis of surface hydrocarbons of workers ( $N=5$ per colony) from experimental colonies $D(\square, \boldsymbol{\square}), E(O, \mathbf{0})$ and $F(\Delta, \mathbf{\Delta})$ before and after the comb swap (b, $a$, respectively). Open symbols represent the chemical profile of bees before the comb swap; closed symbols represent those after the swap. Group centroids are represented by the same symbols as those used for the data points but are larger and are connected by arrows (with dotted lines before the comb swap). The distance between group centroids represents the squared Mahalanobis distance (see Fig. 5). The percentage of the variance explained by each of the two main functions is given in parentheses.

between the bees in the guards' colony and the introduced bees' colony. Larger chemical differences resulted in significantly greater rejection.

In the honeybee, recognition of conspecifics at the adult stage does not appear to be based on heritable cues expressed at the level of individual workers or foodderived odours (Downs \& Ratnieks 1999; Downs et al. 2001). Our chemical analysis was based on the variation in the relative proportions of cuticular hydrocarbons. These chemicals have been shown to be essential for nestmate recognition in many social insects (reviewed in Howard \& Blomquist 2005), but in the case of the honeybee, the role of hydrocarbons is more controversial because fatty acids are also thought to be important recognition cues (e.g. Breed et al. 2004a). However, there is evidence that hydrocarbon profiles differ significantly between subfamilies in a bee hive (Arnold et al. 1996) and honeybee workers treated with additional naturally occurring cuticular hydrocarbons (especially alkenes) are rejected by their own colony (Dani et al. 2005). In addition, several cuticular alkenes are well discriminated by honeybees in proboscis extension conditioning experiments (Châline et al. 2005). Finally, it is well established that hydrocarbons, together with esters, represent an important fraction of comb wax (Fröhlich et al. 2000).

Our study emphasizes the importance of wax comb not only for the ontogeny of recognition cues (e.g. Breed 1998; Breed et al. 2004a), but also for nestmate recognition of forager-aged bees. Two important novel aspects of our study are that we used natural bioassays involving natural guards and forager bees and we manipulated the whole colony by swapping combs. In addition, we carried out chemical analyses which showed that swapping 


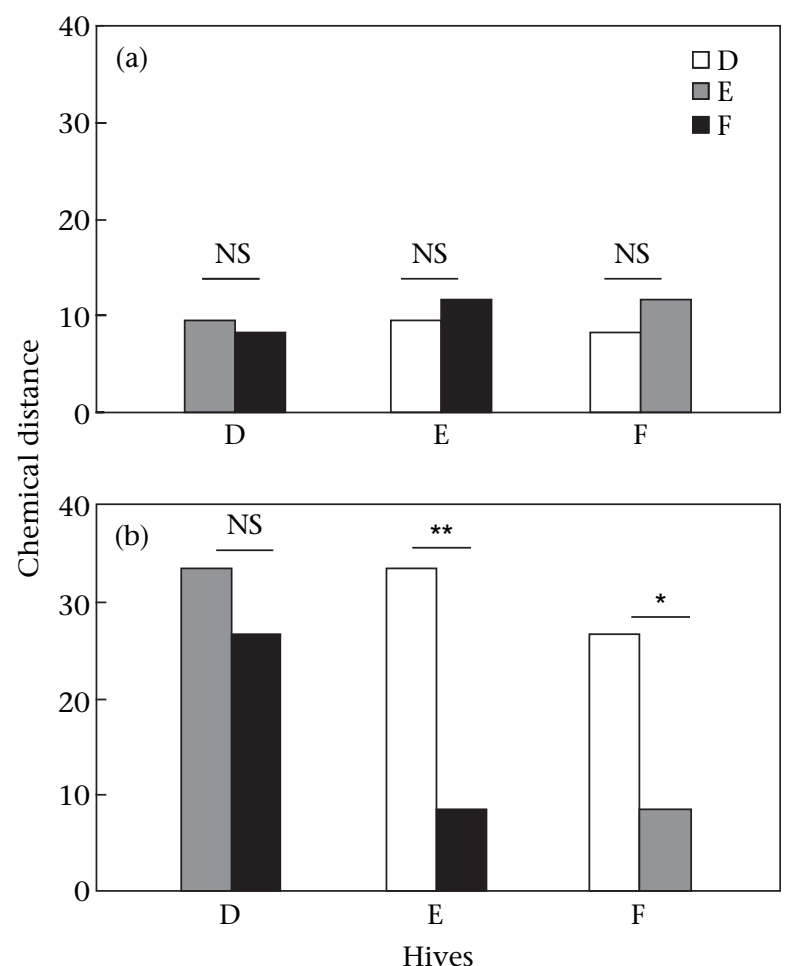

Figure 4. Chemical distances between colonies (squared Mahalanobis distances between group centroids) (a) before and (b) after the comb swap (referring to the discriminant analysis of Fig. 3 ). ${ }^{*} P<0.05$; **P $P$ 0.01: Mann-Whitney $U$ test.

combs increased the chemical similarity of workers from different hives, and that greater chemical similarity is correlated with greater acceptance by guards. As such, our study provides important new information on the effect of comb waxes on honeybee nestmate recognition and more generally in one of the most widely studied model systems for studying recognition.

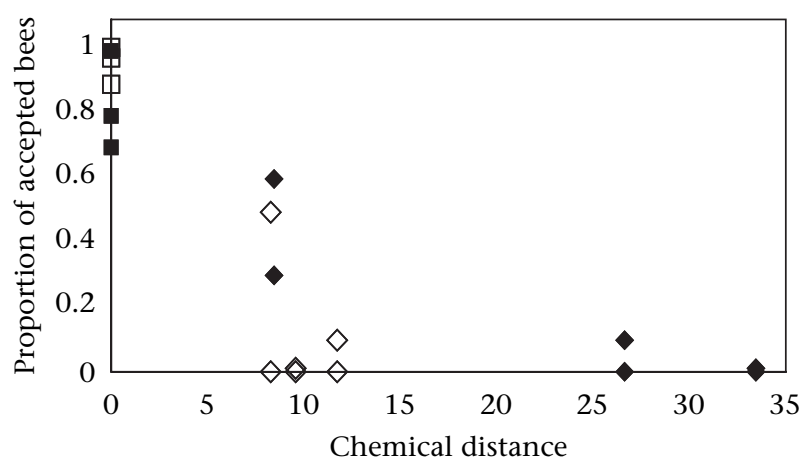

Figure 5. Proportions of introduced forager bees accepted by guards versus the chemical profile distance between the introduced bees' colony and the guards' colony (squared Mahalanobis distance in a discriminant analysis). Each point corresponds to the acceptance data obtained on the same day that forager bees were collected for chemical analysis. $\square, \mathbf{\square}$ : Data from the interaction of guards with nestmates ( $\square$ : before the comb swap; $\mathbf{\square}$ : after the comb swap) have a chemical difference of zero as the guards and introduced bees both came from the same colony. $\diamond, \diamond$ : Data from interactions of guards with non-nestmates ( $\diamond$ : before the swap; $\diamond$ : after the swap).
The acceptance of swapped non-nestmates may be a consequence of a change in the chemical signature of individual bees, which renew their label by being in contact with allocolonial combs, or of a change in the internal template of guard bees, to which the label of an incoming bee is compared, or both. Further studies are needed to uncouple these two effects. Honeybee workers are able to adopt a new template (Breed et al. 2004b). A flexible recognition system is of adaptive value, because changes in label and/or template would be easily reversible and may change naturally. In our study, the effect of the comb swap disappeared several weeks later, with guards resuming correct discrimination between nestmates and non-nestmates.

\section{Acknowledgments}

Research was supported by the 'INSECTS' network of the Universities of Copenhagen, Firenze, Keele, Lausanne, Oulu, Regensburg, Sheffield and the ETH Zurich, financed by the European Commission via the Research Training Network established under the Improving Human Potential Programme (2001-2004; contract CT-2000-00052).

\section{References}

Arnold, G., Quenet, B., Cornuet, J. M., Masson, C., de Schepper, B., Estoup, A. \& Gasqui, P. 1996. Kin recognition in honeybees. Nature, 379, 498.

Beekman, M., Calis, J. N. M., Oldroyd, B. P. \& Ratnieks, F. L. W. 2002. When do honey bee guards reject their former nestmates after swarming? Insectes Sociaux, 49, 56-61.

Breed, M. D. 1998. Chemical cues in kin recognition: criteria for identification, experimental approaches, and the honey bee as an example. In: Pheromone Communication in Social Insects. Ants, Wasps, Bees and Termites (Ed. by R. K. Vander Meer, M. D. Breed, K. Espelie \& M. L. Winston), pp. 57-78. Boulder, Colorado: Westview Press.

Breed, M. D., Garry, M. F., Pearce, A. N., Bjostad, L., Hibbard, B. \& Page, R. E. 1995. The role of wax comb in honeybee nestmate recognition: genetic effects on comb discrimination, acquisition of comb cues by bees, and passage of cues among individuals. Animal Behaviour, 50, 489-496.

Breed, M. D., Perry, S. \& Bjostad, L. B. 2004a. Testing the blank slate hypothesis: why honey bee colonies accept young bees. Insectes Sociaux, 51, 12-16.

Breed, M. D., Diaz, P. H. \& Lucero, K. D. 2004b. Olfactory information processing in honeybee, Apis mellifera, nestmate recognition. Animal Behaviour, 68, 921-928.

Cervo, R. \& Lorenzi, M. C. 1996. Behaviour in usurpers and joiners of Polistes biglumis bimaculatus (Hymenoptera Vespidae). Insectes Sociaux, 43, 255-266.

Châline, N., Sandoz, J.-C., Stephen, J., Martin, S. J., Ratnieks, F. L. W. \& Jones, G. R. 2005. Learning and discrimination of individual cuticular hydrocarbons by honeybees (Apis mellifera). Chemical Senses, 30, 327-335.

Crozier, R. H. \& Pamilo, P. 1996. Evolution of Social Insects. Oxford: Oxford University Press.

Dani, F. R., Corsi, S., Pradella, D., Jones, G. R. \& Turillazzi, S. 2004. Gc-MS analysis of the epicuticule lipids of Apis mellifera reared in central Italy. Insect Social Life, 5, 103-109.

Dani, F. R., Jones, G. R., Corsi, S., Beard, R., Pradella, D. \& Turillazzi, S. 2005. Nestmate recognition cues in the honey bee: differential 
importance of cuticular alkanes and alkenes. Chemical Senses, 30, 477489.

Downs, S. G. \& Ratnieks, F. L. W. 1999. Recognition of conspecifics by honeybee guards uses nonheritable cues acquired in the adult stage. Animal Behaviour, 58, 643-648.

Downs, S. G. \& Ratnieks, F. L. W. 2000. Adaptive shifts in honey bee (Apis mellifera L.) guarding behavior support predictions of the acceptance threshold model. Behavioral Ecology, 11, 326-333.

Downs, S. G., Ratinieks, F. L. W., Badcock, N. S. \& Mynott, A. 2001. Honeybee guards do not use food-derived odors to recognize non-nest mates: a test of the Odor Convergence hypothesis. Behavioral Ecology, 12, 47-50.

Fröhlich, B., Tautz, J. \& Riederer, M. 2000. Chemometric classification of comb and cuticular waxes of the honeybee Apis mellifera carnica. Journal of Chemical Ecology, 26, 123-137.

Gamboa, G. J. 2004. Kin recognition in eusocial wasps. Annales Zoologici Fennici, 41, 789-808.

Howard, R. W. \& Blomquist, G. J. 2005. Ecological, behavioural, and biochemical aspects of insect hydrocarbons. Annual Review of Entomology, 50, 371-393.
Martin, C., Salvy, M., Provost, E., Bagneres, A. G., Roux, M., Crauser, D., Clement, J. L. \& Le Conte, Y. 2001. Variations in chemical mimicry by the ectoparasite mite Varroa jacobsoni according to the developmental stage of the host honey-bee Apis mellifera. Insect Biochemistry and Molecular Biology, 31, 365-379.

Neter, J., Wasserman, W. \& Kutner, M. H. 1996. Applied Linear Statistical Models. Chicago: McGraw-Hill.

Page, R. E., Metcalf, R. A., Metcalf, R. L., Erickson, E. H. \& Lampman, R. L. 1991. Extractable hydro-carbons and kin recognition in honeybee (Apis mellifera L.). Journal of Chemical Ecology, 17, 745-756.

Reeve, H. K. 1989. The evolution of conspecific acceptance thresholds. American Naturalist, 133, 407-435.

Sherman, P. W., Reeve, H. K. \& Pfenning, D. W. 1997. Recognition systems. In: Behavioural Ecology: An Evolutionary Approach (Ed. by J. R. Krebs \& N. B. Davies), pp. 69-96. Oxford: Blackwell Scientific.

Wakonigg, G., Eveleigh, L., Arnold, G. \& Crailsheim, K. 2000. Ontogeny of the cuticular hydrocarbon profiles of honeybee drones. Journal of Apicultural Research, 39, 137-141. 